\title{
Documenting the Public Health Impacts of the Serial Hurricanes of 2017
}

\author{
James J. James, MD, DrPH; José F. Cordero, MD, MPH
}

$\mathrm{F}$ ollowing the devastating serial Atlantic Hurricanes in 2017, the Society for Disaster Medicine and Public Health, Inc. (SDMPH) received a grant from the Robert Wood Johnson Foundation (RWJF) to publish a set of peer-reviewed scientific papers on the medical and public health impacts of the storms. It was hoped to collect enough articles to populate a full issue of our journal, Disaster Medicine and Public Health Preparedness (DMPHP). The call for papers was open-ended as to duration but there was a strong emphasis on timing in an effort to publish material that could inform the ongoing recovery process.

Because Puerto Rico was so devastated by the hurricanes, the unprecedented destruction of Maria exacerbating damage from Irma, a special effort was made to collect reports from a broad array of individuals actively involved in the response from across the many sectors involved in providing health security for the population of Puerto Rico. We quickly realized that, although there was a large and continued disaster response from external sources, there was also a tremendous internal response, notably from the health and public health systems, but also from literally every component of the public and private sectors within Puerto Rico, and, most importantly, from the Puerto Rican people.

To better capture these myriad experiences and the conclusions and recommendations emanating from them, it was felt that a journal workshop should be held in Puerto Rico to encourage the translation of the ongoing response and recovery efforts into sciencebased recommendations through peer review publication. This concept was discussed with Dr. José Cordero, a distinguished physician and Public Health Professor of Puerto Rican descent at the University of Georgia, who was one of the first professionals from the continental US to respond to the ongoing public health crises post-Maria. Dr. Cordero strongly embraced the idea and worked with the Puerto Rico Science, Technology and Research Trust to set up the workshop held in March of 2018.
The event proved to be a great success. Over 20 Puerto Rican health and public health leaders and scientists were not only in attendance but obviously excited by our proposals and committed themselves to our undertaking. The RWJF Workshop went on to produce two notable outcomes: 1) the number of manuscripts eventually received under the original call for papers was sufficient to populate not one, but two full journal issues, and 2) as a result of discussions at the workshop, the concept of presenting our efforts and those of others at a summit was conceived. This concept gained further impetus as the Trust had been recently selected under a CDC grant to house a Hurricane Hub and join a network of like-funded centers in other US cities and a summit would provide the opportunity to collect, analyze, integrate and act on recommendations from the many sectors that had participated in the response and ongoing recovery efforts to the serial hurricanes.

The concept of a summit quickly materialized into an agreement between the Trust and SDMPH to partner in sponsoring the event, with the Trust providing the necessary guarantees to support such an endeavor. The stated goal of the event would be the enumeration and publication of science-based recommendations from the overall body of work to be collected in conjunction with our efforts and those of others involved in response and recovery efforts, and to support development of an integrated community resilience model. The Caribbean Strong Summit was held in February 2019 in San Juan and was a tremendous success with over 500 attendees. Further details and the summit agenda can be accessed at https://prsciencetrust.org/ caribbeanstrong/. The overall conduct of the summit, the enumeration and analysis of recommendations, and the resulting resilience model will be described in a forthcoming publication.

As to the articles collected under the RWJF grant, the first half were published in the February 2019 issue of DMPHP and have been extremely well received. This, the second serial hurricane issue, closes out the articles collected under the grant and has been made possible with further support from RWJF and the Trust. The 
ability to consolidate all of our work in this second hurricane issue is important as the Trust, with its imbedded Hurricane Hub, is interested in sponsoring a follow-on summit to Caribbean Strong focusing on the outcomes from the first meeting, presenting the resiliency model resulting from the first, and, more importantly, highlighting their continuing efforts to insure a Resilient Caribbean. The importance of this work has been tragically underscored by the ongoing series of earthquakes being experienced in Puerto Rico as this issue of DMPHP is being finalized. 